\title{
Is Total Hip Arthroplasty a Cost-Effective Option for Management of Displaced Femoral Neck Fractures? A Trial-Based Analysis of the HEALTH Study
}

\author{
Daniel Axelrod, MD, MSc (Cand), ${ }^{a}$ Jean-Éric Tarride, PhD, ${ }^{\text {b.c.d }}$ Seper Ekhtiari, MD, ${ }^{a}$ \\ Gordon Blackhouse, MBA, MSc, ${ }^{b, c}$ Herman Johal, MD, MPH, FRCSC, ${ }^{a}$ Sofia Bzovsky, MSc, ${ }^{a}$ \\ Emil H. Schemitsch, MD, FRCSC, ${ }^{e}$ Diane Heels-Ansdell, MSc, ${ }^{a}$ Mohit Bhandari, MD, PhD, FRCSC, ${ }^{a, b}$ \\ and Sheila Sprague, $P h D^{a, b}$ on behalf of the HEALTH Investigators
}

\begin{abstract}
Background: Displaced femoral neck fractures are a significant source of morbidity and mortality and can be treated with either hemiarthroplasty (HA) or total hip arthroplasty (THA). Proponents of THA have argued THA offers lower risk of revision, with improved functional outcomes when compared to HA. To evaluate cost effectiveness of THA compared with HA, a trial-based economic analysis of the HEALTH study was undertaken.
\end{abstract}

Methods: Health care resource utilization (HRU) and health-related quality of life (HRQoL) data were collected postoperatively and costed using publicly available databases. Using EuroQol-5 Dimensions (EQ-5D) scores, we derived quality adjusted life years (QALYs). A $1.5 \%$ discount rate to both costs and QALYs was applied. Age analyses per age group were conducted. All costs are reported in 2019 Canadian dollars.

Results: When compared with HA, THA was not cost-effective for all patients with displaced femoral neck fractures (\$150,000/QALY gained). If decision makers were willing to spend $\$ 50,000$ or
$\$ 100,000$ to gain one QALY, the probability of THA being costeffective was $12.8 \%$ and $32.8 \%$, respectively. In a subgroup of patients younger than 73 (first quartile), THA was both more effective and less costly. Otherwise, THA was more expensive and yielded marginal HRQoL gains.

Conclusions: Our results suggest that for most patients, THA is not a cost-effective treatment for displaced femoral neck fracture management versus HA. However, THA may be cost effective for younger patients. These patients experience more meaningful improvements in quality of life with less associated cost because of shorter hospital stay and fewer postoperative complications.

Key Words: economic evaluation, displaced femoral neck fracture, total hip arthroplasty, hemiarthroplasty

Level of Evidence: Economic Level II. See Instructions for Authors for a complete description of levels of evidence.

(J Orthop Trauma 2020;34:S37-S41)

Accepted for publication August 11, 2020

From the ${ }^{a}$ Division of Orthopaedic Surgery, Department of Surgery, McMaster University, Hamilton, ON, Canada; ${ }^{b}$ Department of Health Research Methods, Evidence, and Impact, McMaster University, Hamilton, ON, Canada; ${ }^{\circ}$ Center for Health Economics and Policy Analysis (CHEPA), McMaster University, and Programs for Assessment of Technology in Health (PATH), The Research Institute of St. Joe's Hamilton, St. Joseph's Healthcare Hamilton, Hamilton, ON, Canada; ${ }^{\mathrm{d}}$ McMaster Chair in Health Technology Management Hamilton, Hamilton, ON, Canada; and ${ }^{\mathrm{e}}$ Department of Surgery, University of Western Ontario, London, ON, Canada.

The HEALTH trial was supported by research grants from the Canadian Institutes of Health Research (CIHR) (MCT-90168), National Institutes of Health (NIH) (1UM1 AR063386-01), ZorgOnderzoek Nederland-medische wetensehappen (ZonMw) (17088.2503), Sophies Minde Foundation for Orthopaedic Research, McMaster Surgical Associates, and Stryker Orthopaedics. The funding sources had no role in design or conduct of the study; the collection, management, analysis, or interpretation of the data; or the preparation, review, or approval of the manuscript.

H. Johal reports board or committee member for the Orthopaedic Research Society, outside the submitted work. E. H. Schemitsch reports personal fees from Acumed, LLC, personal fees from Amgen Co, research support from Biocomposites, board or committee member for the Canadian Orthopaedic Association, personal fees from DePuy, board or committee member for the Hip Society, board or committee member for the International Society for Fracture Repair, personal fees from ITS, editorial or governing board for the Journal of Orthopaedic Trauma, board or committee member for the Orthopaedic Trauma Association, editorial or governing board for the Orthopaedic Trauma Association International, board or committee member for the Osteosynthesis and Trauma Care Foundation, personal fees from Pentopharm, personal fees from Sanofi-Aventis, personal fees from Saunders/Mosby-Elsevier, personal fees from Smith \& Nephew, personal fees from Springer, personal fees from Stryker, personal fees from Swemac, and personal fees from Zimmer, outside the submitted work. M. Bhandari reports research support from Acumed, LLC, research support from Aphria, research support from Ferring Pharmaceuticals, research support and personal fees from Pendopharma, and research support and personal fees from Sanofi-Aventis, outside the submitted work. S. Sprague reports editorial or governing board for BMS Women's Health, employment from Global Research Solutions Inc, and employment from McMaster University, outside the submitted work. The remaining authors report no conflict of interest.

Supplemental digital content is available for this article. Direct URL citations appear in the printed text and are provided in the HTML and PDF versions of this article on the journal's Web site (www.jorthotrauma.com).

Reprints: Daniel Axelrod, MD, MSc (Cand.), Division of Orthopaedic Surgery, Department of Surgery, McMaster University, 293 Wellington St. N, Suite 110, Hamilton, ON L8L 8E7, Canada (e-mail: Daniel.axelrod@medportal.ca).

Copyright (C) 2020 Wolters Kluwer Health, Inc. All rights reserved.

DOI: 10.1097/BOT.0000000000001932 


\section{INTRODUCTION}

Hip fractures are the most common injury suffered by the elderly and contribute substantial morbidity to these patients, who often have multiple pre-existing comorbidities and are frail. ${ }^{1}$ Moreover, the mortality associated with hip fractures approaches $25 \%$ for all patients. ${ }^{2}$ The burden of these injuries - in both human and economic terms - is substantial. Up to 10 percent of patients with these injuries will require a secondary operation, thus multiplying the economic impact of these injuries. ${ }^{3}$ These revision surgeries range from 2 to 3 and half times more costly than the primary procedure. ${ }^{3}$

For displaced femoral neck fractures, both partial joint replacement of the hip (hemiarthroplasty, HA) and total joint replacement [total hip arthroplasty (THA)], are considered standard of care in the elderly. ${ }^{4}$ Those who have argued for HA suggest that it represents a less morbid, less expensive and noninferior treatment option to THA. Those who argue for THA believe it yields fewer reoperations, and affords better quality of life, similar to patients who receive THA for osteoarthritis. ${ }^{5}$

A recent meta-analysis suggested that THA for femoral neck fractures did meaningfully improve quality of life for patients while reducing unplanned reoperations. ${ }^{4}$ However, the meta-analysis was dependent on small, underpowered clinical trials with non-standardized adjudication of outcomes. Moreover, issues of confounding due to expertise bias were pervasive in all studies. Ultimately, even after multiple studies and meta-analyses, ${ }^{3,4,6}$ there remains uncertainty regarding the best treatment for displaced femoral neck fractures in the elderly.

To address this issue, a multi-national, multi-center expertise-based randomized controlled trial termed the Hip Fracture Evaluation With Alternatives of Total Hip Arthroplasty versus Hemi-Arthroplasty (HEALTH) trial $^{7}$ was performed. The HEALTH study did not find any clinically meaningful differences in either rate of unplanned reoperations or health utility when comparing THA to HA. At the same time that the main trial was undertaken, relevant health care utilization data was also collected to facilitate an economic evaluation to inform health care policy leaders and key stakeholders in this domain. In this study, we report on the results of these economic findings through a trial based economic analysis.

\section{METHODS}

\section{Study Design and Participants}

The study design and clinical results of the HEALTH trial are described elsewhere. ${ }^{7}$ Health care resource utilization and health-related quality of life [EuroQol Five-Dimension (EQ-5D)] information were collected during follow-up visits at $6,12,24,38$, and 52 weeks, and at 18 and 24 months.

\section{Health Care Resource Utilization}

A case report form was completed for each follow-up visit to collect information on health-related quality of life, secondary procedures, serious adverse events, physiotherapy visits and hospital readmissions. As the frequency of care was not captured for physiotherapy, we assumed that patients would use 7 physiotherapy visits.

The unit costs of secondary procedures were derived from a patient cost estimator from the Canadian Institute of Health Information. ${ }^{8}$ The patient cost estimator is an initiative which is informed by costing data submitted from all provinces across Canada, with the exception of Quebec. In addition, costs can be specified by province and by patient age. For the purpose of this analysis, all cost estimates were taken from the perspective of the Ontario health care system. Unit costs of implants were taken from costing inventory at Hamilton General Hospital in Hamilton, Ontario.

We assumed the hourly rate for provincially covered physiotherapy was $\$ 120.9^{9}$ Unit costs used in this economic evaluation are presented in Supplemental Digital Content 1 (see Appendix 1, http://links.lww.com/JOT/B211). All costs are stated in 2019 Canadian dollars.

\section{Health-Related Quality of Life and QALYs}

Although both the Short Form-12 (SF-12) and the EQ5D-5L questionnaires were collected in the HEALTH study, the economic results were derived from the EQ-5D, as this instrument most easily allows the calculation of a health utility score, necessary to calculate quality-adjusted life years (QALYs) and is validated for use in this population. ${ }^{10}$ QALYs combine quantity of life with health-related quality of life and are used in cost-utility analyses to compare outcomes between interventions.

The EQ-5D measures health-related quality of life in 5 dimensions (mobility, self-care, usual activities, pain/ discomfort, and anxiety/depression) with 3 levels of scoring per attribute. Using a scoring algorithm, the individual responses to each of the domains are transformed into a health utility score where 0 represents death and 1 the best imaginable health state. The minimal important difference for the EQ-5D was set at 0.05. ${ }^{11}$ QALYs were calculated for each intervention by weighting the utility scores by time spent in health states using an area under the curve approach.

\section{Cost-Effectiveness Analyses}

Utilizing a trial-based cost-effectiveness approach following good practice guidelines, ${ }^{12,13}$ use of THA versus HA was assessed in incremental cost per QALY gained. Overall costs and outcomes were first evaluated to determine if either strategy was dominant (both less costly and better quality of life). If neither strategy was found to be dominant, incremental cost-effectiveness ratios (ICERs) were estimated by calculating the difference in cost between THA and HA divided by the difference in their effect (ie, QALYs). Uncertainty regarding costs and effects due to sampling variability associated with the trial was measured using nonparametric bootstrapping techniques. ${ }^{14}$

The bootstrapping technique entails drawing a random sample from the original dataset (with replacement) and then calculating the mean costs and effects associated with each treatment group (ie, THA and HA). The sampling process was repeated 2000 times to create a sampling distribution and generate means, SEs, and 95\% confidence intervals (CIs) for the incremental costs, incremental QALYs, and ICERs. Cost- 
TABLE 1. Average Cost per Patient (Can \$2019)

\begin{tabular}{|c|c|c|c|c|}
\hline & \multirow{3}{*}{$\begin{array}{c}\text { THA } \\
(\mathbf{N}=\mathbf{7 1 8}) \\
\text { Mean (SE) }\end{array}$} & \multirow{3}{*}{$\begin{array}{c}\text { HA } \\
(\mathrm{N}=\mathbf{7 2 3}) \\
\text { Mean (SE) }\end{array}$} & \multicolumn{2}{|l|}{ Difference } \\
\hline & & & & \\
\hline & & & Mean $(95 \%$ CI) & $\boldsymbol{P}$ \\
\hline$\overline{\text { Device }}$ & $\$ 1789$ & $\$ 925$ & & \\
\hline Initial procedure cost & $\$ 16,756(\$ 1220)$ & $\$ 15,732(\$ 840)$ & $\$ 1024(-\$ 1880$ to $\$ 3928)$ & 0.10 \\
\hline Physiotherapy & $\$ 592(\$ 10.3)$ & $\$ 596(\$ 10.1)$ & $\$ 4(-\$ 23$ to $\$ 33)$ & 0.74 \\
\hline Costs from adverse events & $\$ 9469(\$ 1100)$ & $\$ 7933$ (\$919) & $\$ 1536(-\$ 1274$ to $\$ 4346)$ & 0.28 \\
\hline Costs from second procedures & $\$ 4231(\$ 1083)$ & $\$ 2541(\$ 526)$ & $\$ 1690(-\$ 666$ to $\$ 4047)$ & 0.16 \\
\hline Payer perspective & $\$ 32,851(\$ 2368)$ & $\$ 27,358(\$ 1513)$ & $\$ 5493(-\$ 14$ to $\$ 11,000)$ & 0.06 \\
\hline
\end{tabular}

This represents the average overall costs attributed to each patient by each cost category, though most patients did not suffer from adverse events or undergo second procedures.

effectiveness acceptability curves (CEACs) were used to present the probability of THA to be cost effective at different willingness-to-pay thresholds (ie, \$50,000/QALY gained or $\$ 100,000 /$ QALY gained-2 commonly cited costeffectiveness thresholds) while taking into account the uncertainty associated with the trial data. ${ }^{15}$ Analyses were conducted from a public payer and societal perspective over a 2-year time horizon. Both costs and QALYs collected after one year were discounted at $1.5 \%$ per year, as per Canadian Agency for Drug and Technologies in Health (CADTH) guidelines, to indicate that either a single QALY or amount of cost was more valuable now than in one year's time. ${ }^{16}$

\section{Sensitivity and Subgroup Analyses}

Multiple sensitivity analyses were planned. First, we planned to assess the impact of change in discount rate, varying from $0 \%$ to $1.5 \%$ (base case) to $3 \%$. In addition, we performed a sensitivity analysis evaluating the difference in ICERs using a multiple imputation model (base case) and listcase deletion. Moreover, a sensitivity analysis was planned to modify the cost of the technology (THA) by 30\% in either direction, as recommended by CADTH. ${ }^{16}$ Finally, we performed a sensitivity analysis with changing mortality rates per group if mortality rates were found to be meaningfully different between THA and HA. We also performed a posthoc subgroup analysis by age quartile, hypothesizing that THA would be most cost effective for younger quartiles. This analysis mirrors a similar age stratified analysis performed for the main HEALTH trial.

\section{RESULTS}

Similar to the results of the clinical trial, there were no clinically meaningful differences between the 2 treatment groups in unplanned reoperation or health utility (Table 1).

The costs from the single payer perspective were estimated to be $\$ 32,851$ per patient $(\mathrm{SE}=\$ 2368)$ for THA and $\$ 27,358(\mathrm{SE}=\$ 2781)$ for $\mathrm{HA}$. The total number of QALYs estimated using the EQ-5D score over the two-year time horizon was $1.40(\mathrm{SE}=0.02)$ for THA and $1.36(\mathrm{SE}=$ $0.019)$ for HA. The difference of 0.04 QALYs $(95 \% \mathrm{CI}$ : -0.01 to 0.11 ) was not significantly different. The resulting ICER of THA compared with HA for the treatment of patients with displaced femoral neck fractures was calculated at $\$ 151,640 /$ QALY gained (Fig. 1). Table 2 presents these results.

Our sensitivity analyses produced similar results. When either a $0 \%$ or $3 \%$ discount rate was applied to both costs and QALYs at one year, the ICERs did not meaningfully change. Finally, when comparing imputed to not imputed EQ-5D values, EQ-5D scores each decreased by 0.01 points for both THA and HA, yielding no change in the difference in EQ-5D scores. In addition, neither decreasing nor increasing the cost of THA by $30 \%$ yielded meaningful differences in ICERs, because implant costs only represented $10 \%$ of total costs per patient.

Finally, a subgroup analysis by age quartile was performed. For patients younger than 73 (first quartile), THA was both less expensive (-\$3693) and provided more health utility (0.10 QALYs). In contrast, for all other age quartiles, HA provided similar utility at a reduced cost (Table 3).

The CEACs curves presented in Fig. 2 indicate that when compared with HA, the probability of THA being cost-effective for treating operatively managed displaced femoral neck fractures was $12.8 \%$ and $32.8 \%$, if decision makers

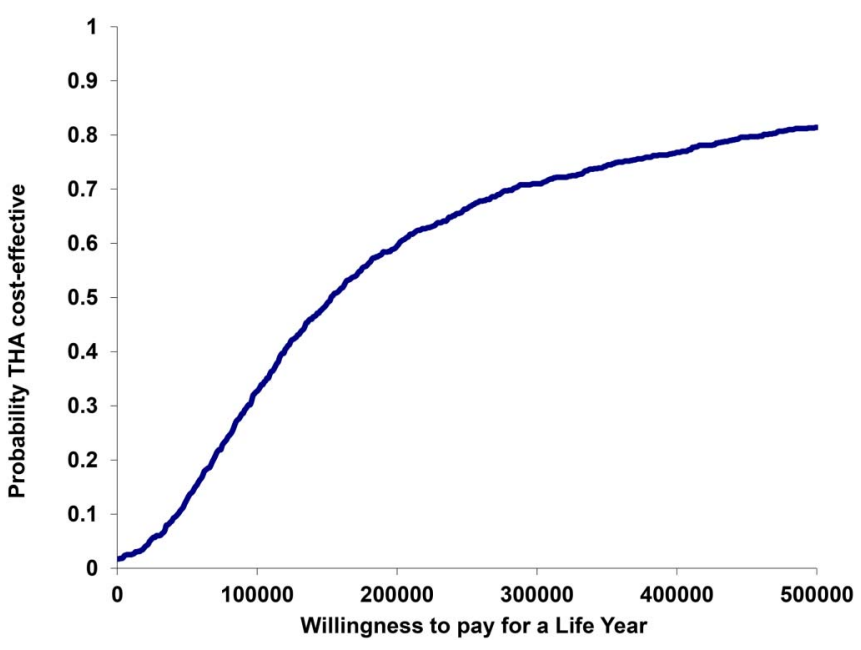

FIGURE 1. Cost-effectiveness plane. Editor's Note: A color image accompanies the online version of this article.

www.jorthotrauma.com | S39 
TABLE 2. Cost Effectiveness Analysis

\begin{tabular}{|c|c|c|c|c|c|c|c|}
\hline & Cost & Iean $(95 \% \mathrm{CI})$ & & & ALYs Mean & $\%$ CI) & ICER \\
\hline & THA & HA & $\begin{array}{c}\Delta \\
\text { Cost }\end{array}$ & THA & HA & $\Delta$ QALYs & $\begin{array}{c}\text { \$/QALY } \\
\text { Gained }\end{array}$ \\
\hline $\begin{array}{l}\text { All patients } \\
(\mathrm{N}=1441)\end{array}$ & $\$ 32,851(\$ 5275-\$ 194,033)$ & $\$ 27,358(\$ 5211-\$ 144,953)$ & $\$ 5493$ & $1.40(0-1.97)$ & $1.36(0-1.97)$ & $0.04(-0.01$ to 0.09$)$ & $\$ 151,640$ \\
\hline
\end{tabular}

Note that bootstrap sampling data is different than raw trial data and produces marginally different estimates to account for uncertainty.

were willing to spend $\$ 50,000$ or $\$ 100,000$ to gain one QALY, respectively.

\section{DISCUSSION}

To the authors knowledge, this is the most up-to-date contemporary trial-based economic evaluation comparing THA versus HA in elderly patients with displaced femoral neck fractures. Previous studies have concluded, similar to our study, that THA is likely not a cost effective option in the majority of patients who suffer femoral neck fractures. ${ }^{17,18}$ Consistent with the results of the HEALTH trial and other systematic reviews, we did not observe any clinically significant differences in either reoperation or health-related quality of life by treatment group. Moreover, through a methodologically rigorous trial-based evaluation, we were able to assess whether or not THA was a costeffective intervention and under what circumstances the treatment may be cost acceptable.

At both $\$ 50,000$ and $\$ 100,000$ willingness to pay thresholds, our cost effectiveness analysis revealed that compared with HA, THA is not likely to be a cost-effective intervention for all patients with displaced femoral neck fractures. Moreover, in no sensitivity analysis did THA represent a cost-effective intervention. However, in subgroup analysis, for patients younger than 73 (the youngest quartile), THA was a cost-effective intervention, because they received more QALYs for less estimated cost. In contrast, for all other age quartiles, THA was more expensive and did not provide more quality of life. Younger patients may be able to mobilize quicker with a THA as compared to the eldest cohort, reducing the comparative additional cost to HA. Moreover, younger patients may notice and appreciate the increased mobility offered from a THA, leading to higher health-related quality of life.

Although previous economic evaluations have been conducted comparing THA to HA, these were either performed using model approaches with significant assumptions or trial-based approaches using small trials. ${ }^{18,19}$ In a recent meta-analysis including the HEALTH trial, the HEALTH trial contributed more than $50 \%$ of all patients to ever be evaluated in a randomized controlled trial comparing THA to HA. ${ }^{5}$ The dominance of this trial, in both sample size and methodologic rigor, necessitates that it be evaluated as a separate entity and through a single trial-based analysis.

Our study has a number of strengths and some limitations. As described above, the primary strength is that our evaluation is based on a multi-national, rigorouslyconducted prospective randomized controlled trial which is the largest ever in its field. As is true of all trial-based analyses, using measured outcomes from patients rather than using modeling practices reduces the number of assumptions on both costs and utility. In addition, through using a standard discount rate, performing bootstrapping techniques for sampling uncertainty, and conducting multiple sensitivity analyses determined a priori, we have adhered to good practice guidelines for trial based economic evaluations. ${ }^{13,16}$ In addition, the use of CEACs easily illustrate the likelihood of THA to be cost-effective at given willingness to pay thresholds.

A notable limitation of our economic evaluation is that cost and health-related quality of life data were not available for all patients. Even though EQ-5D scores were absent for only approximately $8 \%$ of observations, relevant cost data such as medication use and return to work parameters were not collected throughout the trial. For missing health-related quality of life data, multiple imputation techniques were used to generate complete data sets, and subsequent sensitivity analyses were performed to generate confidence in these estimates. Regarding return to work, the mean age of patients enrolled in this trial was 79 years ( \pm 8 years) and thus patients would be unlikely to be employed before their injury and to return to work, regardless of intervention. ${ }^{20}$ Moreover, costing data, in particular for orthopedic secondary procedures, was often not sufficiently specific (eg, all revisions without infections were costed the same). However, the major cost driver for these events was length of stay during hospital readmission and, therefore, the severity of the event was more

TABLE 3. Subgroup Analysis of Cost Effectiveness by Age Quartile

\begin{tabular}{|c|c|c|c|c|c|c|c|}
\hline & \multicolumn{3}{|c|}{ Cost Mean } & \multicolumn{3}{|c|}{ QALYs Mean } & \multirow[b]{2}{*}{ ICER \$/QALY Gained } \\
\hline & THA & HA & $\Delta$ Cost & THA & HA & $\overline{\Delta \text { QALYs }}$ & \\
\hline Age $<73$ & $\$ 24,156$ & $\$ 27,849$ & $-\$ 3693$ & 1.54 & 1.44 & 0.10 & $-\$ 38,175$ (dominated) \\
\hline Age $73-79$ & $\$ 42,441$ & $\$ 26,500$ & $\$ 15,941$ & 1.48 & 1.45 & 0.03 & $\$ 574,220$ \\
\hline Age $80-84$ & $\$ 32,709$ & $\$ 21,947$ & $\$ 10,762$ & 1.31 & 1.32 & -0.01 & $-\$ 789,066$ (dominated) \\
\hline Age 85 and older & $\$ 31,918$ & $\$ 31,978$ & $\$-60$ & 1.28 & 1.22 & 0.06 & -\$1072 (dominated) \\
\hline
\end{tabular}




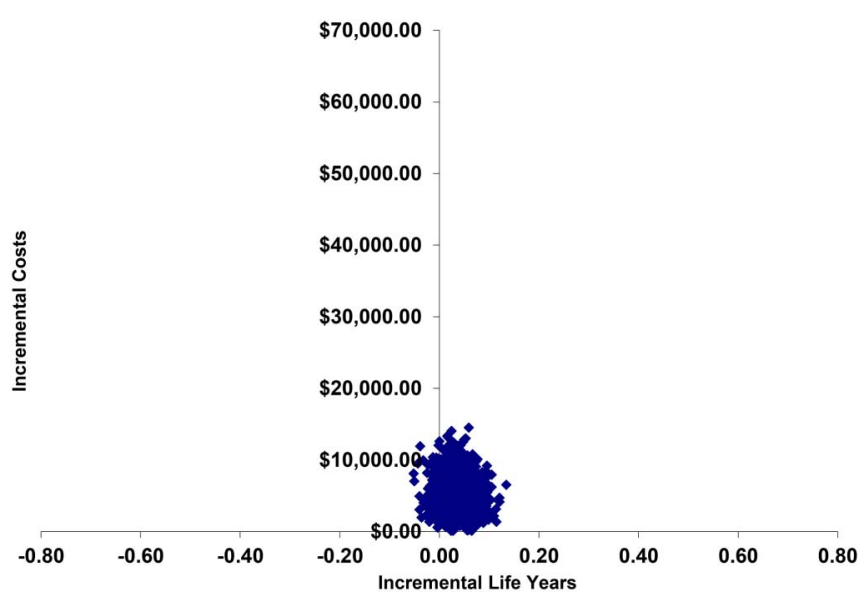

FIGURE 2. Cost-effectiveness acceptability curves. Editor's Note: A color image accompanies the online version of this article.

accurately captured in costing analysis. Another limitation of this analysis is the 2-year duration of the HEALTH study. It is possible that with 2 years of follow-up, all possible costs and outcomes following surgery may not have been accounted for. In particular, it may take over 2 years for acetabular deterioration from a hemiarthroplasty to occur and to require revision. However, the best available data suggest that health outcomes following hip fractures take 2 years to stabilize (including rate of revision operations and functional outcomes). ${ }^{21}$

Our analysis provides further evidence as to the cost effectiveness of THA in the treatment of patients with displaced femoral neck fractures. We found no meaningful differences in health-related quality of life between THA and HA, whereas THA cost over $\$ 5000$ more per patient. However, for the youngest cohort of patients, THA was found to provide meaningful clinical benefit and at less cost. The cost effectiveness of THA for femoral neck fractures should be further evaluated prospectively and may justify reimbursement of the technology for this patient group. The results of this trial, although most applicable to the single payer health care system in Ontario, Canada, may be useful in informing health policy decisions internationally.

\section{ACKNOWLEDGMENTS}

The authors thank the HEALTH Investigators (http:// links.lww.com/JOT/B235).

\section{REFERENCES}

1. Rapp K, Büchele G, Dreinhöfer K, et al. Epidemiology of hip fractures. $Z$ Gerontol Geriatr. 2019;52:10-16.
2. Veronese N, Maggi S. Epidemiology and social costs of hip fracture. Injury. 2018;49:1458-1460.

3. Reeves RA, Schairer WW, Jevsevar DS. The national burden of periprosthetic hip fractures in the US: costs and risk factors for hospital readmission. Hip Int. 2019;29:550-557.

4. Lewis DP, Wæver D, Thorninger R, et al. Hemiarthroplasty vs total hip arthroplasty for the management of displaced neck of femur fractures: a systematic review and meta-analysis. J Arthroplasty. 2019;34:1837-1843.e2.

5. Ekthiari S, Gormly J, Axelrod D, et al. Total hip arthroplasty versus hemiarthroplasty for displaced femoral neck fracture: a systematic review and meta-analysis of randomized controlled trials. J Bone Joint Surg Am. 2020

6. Carroll C, Stevenson M, Scope A, et al. Hemiarthroplasty and total hip arthroplasty for treating primary intracapsular fracture of the hip: a systematic review and cost-effectiveness analysis. In: NIHR Health Technology Assessment Programme: Executive Summaries. NIHR Journals Library; 2011;102(18),1638-1645.

7. HEALTH Investigators, Bhandari M, Einhorn TA, Guyatt G, et al. Total hip arthroplasty or hemiarthroplasty for hip fracture. N Engl J Med. 2019; 381:2199-2208.

8. Canadian Institute of Health Intitatives. CIHI Patient Cost Estimator [Internet]. 2020. Available at: https://www.cihi.ca/en/patient-costestimator. Accessed March 20, 2020

9. Province of Ontario. Physiotherapy Reimbursement [Internet]. 2019. Available at: http://www.health.gov.on.ca/en/pro/programs/physio/ physio_pro_doctors_nurses.aspx. Accessed March 20, 2020.

10. Parsons N, Griffin XL, Achten J, et al. Outcome assessment after hip fracture: is EQ-5D the answer? Bone Joint Res. 2014;3:69-75.

11. McClure NS, Al Sayah F, Xie F, et al. Instrument-defined estimates of the minimally important difference for $\mathrm{EQ}-5 \mathrm{D}-5 \mathrm{~L}$ index scores. Value Heal. 2017;20:644-650.

12. Ramsey SD, Willke RJ, Glick H, et al. Cost-effectiveness analysis alongside clinical trials II-an ISPOR good research practices task force report. Value Heal. 2015;18:161-172.

13. Husereau D, Drummond M, Petrou S, et al. Consolidated health economic evaluation reporting standards (CHEERS) statement. BMJ. 2013; 346:f1049-1055.

14. Hunink MGM, Bult JR, De Vries J, et al. Uncertainty in decision models analyzing cost-effectiveness: the joint distribution of incremental costs and effectiveness evaluated with a nonparametric bootstrap method. Med Decis Mak. 1998;18:337-346.

15. Woods B, Revill P, Sculpher M, et al. Country-level cost-effectiveness thresholds: initial estimates and the need for further research. Value Heal. 2016;19:929-935.

16. Canadian Agency for Drugs and Technologies in Health. Guidelines for the Economic Evaluation of Health Technologies: Canada. 3rd ed. Ottawa, ON; 2006.

17. Burgers PT, Hoogendoorn M, Van Woensel EAC, et al. Total medical costs of treating femoral neck fracture patients with hemi- or total hip arthroplasty: a cost analysis of a multicenter prospective study. Osteoporos Int. 2016;27:1999-2008.

18. Slover J, Hoffman MV, Malchau H, et al. A cost-effectiveness analysis of the arthroplasty options for displaced femoral neck fractures in the active, healthy, elderly population. J Arthroplasty. 2009;24:854-860.

19. Healy WL, Iorio R. Total hip arthroplasty: optimal treatment for displaced femoral neck fractures in elderly patients. Clin Orthop Relat Res. 2004;429:43-48.

20. Statistics Canada. Labour Force Characteristics by Sex and Detailed Age Group, Annual, Inactive (X 1,000) [Internet]. 2019. Available at: https://www150.statcan. gc.ca/t1/tbl1/en/tv.action?pid=1410001701. Accessed March 15, 2020.

21. Ravikumar KJ, Marsh G. Internal fixation versus hemiarthroplasty versus total hip arthroplasty for displaced subcapital fractures of femur-13 year results of a prospective randomised study. Injury. 2000;31:793-797. 